\title{
Latest results and sensitivities for solar dark matter searches with IceCube
}

\author{
The IceCube Collaboration ${ }^{\dagger}$ \\ $\dagger$ http://icecube.wisc.edu/collaboration/authors/icrc17_icecube \\ E-mail: seongjin.in@gmail.com
}

\begin{abstract}
Dark matter from the Galactic dark matter halo could scatter off of nuclei in the Sun and become gravitationally captured, accumulating in the center. The accumulated dark matter may annihilate into standard model particles and produce energetic neutrinos in sequent decays. Neutrinos with energies below a few hundred $\mathrm{GeV}$ escape the Sun without any significant absorption and can be searched for using a neutrino telescope. IceCube has produced the most stringent bounds on spin-dependent scattering of dark matter with hydrogen by looking for this signal. We present IceCube's latest solar dark matter search results including a new all-neutrino flavor result that substantially improves IceCube's sensitivity for dark matter particles with masses below O(100) GeV.
\end{abstract}

Corresponding authors: Seongjin $\mathrm{In}^{1 *}$ and Klaus Wiebe ${ }^{2}$

${ }^{1}$ Department of Physics, Sungkyunkwan University, Seobu 16419, Suwon, South Korea

${ }^{2}$ Johannes Gutenberg University Mainz, Germany

35th International Cosmic Ray Conference ICRC2017

10-20 July, 2017

Bexco, Busan, Korea

${ }^{*}$ Speaker. 


\section{Introduction}

Dark matter (DM) annihilations in the Sun could result in observable signals at neutrino telescopes. IceCube, the worlds largest neutrino observatory [1], located in South Pole, has searched for such signals from the Sun and we present a status of on-going searches as well as an outlook for future analyses and detector upgrades.

Dark matter (denoted $\chi$ ) from the Galactic dark matter halo can be gravitationally captured by to the Sun after an initial scattering of Hydrogen or heavier nuclei. Dark matter will accumulate in the center of the Sun and is expected to self-annihilate in many popular DM scenarios, such as Weakly Interactive Massive Particles (WIMPs). Under the assumption that DM capture and annihilation have reached equilibrium, the expected neutrino flux is independent of the dark matter self-annihilation cross section and only depends on the scattering process that initiates capture. The equilibrium assumption [2] is reasonable given the age of the Sun and allows for an easy conversion of a neutrino flux to a corresponding WIMP-proton cross section. The expected neutrino energy range of interest for IceCube is from few $\mathrm{GeV}$ to $1 \mathrm{TeV}$, as neutrino signals above $100 \mathrm{GeV}$ start to be attenuated due to absorption in the Sun.

In these proceedings we summarize results using muon neutrinos and three years of IceCube data [3]. We present a new all-flavor search for solar WIMPs [4] and give an outlook in future solar WIMP searches with IceCube and planned upgrades.

\section{Solar Dark Matter annihilation search with 3 years of IceCube data}

Solar Dark Matter annihilation search with IceCube-79strings (IC79) was performed in 2011 and we have updated this analysis with 3 years data with the final 86-string detector configuration (IC86). The sensitivity is better than the previous due to the additional statistics and improved analysis method. As a low energy point-like source analysis, the angular resolution of the detector is important. Therefore, $v_{\mu}$ samples are the main focus of this analysis.

For the signal simulation, we used DarkSUSY [5] and WimpSim [6] to calculate the predicted neutrino signal flux and the propagation to the Earth, taking neutrino oscillations into account.
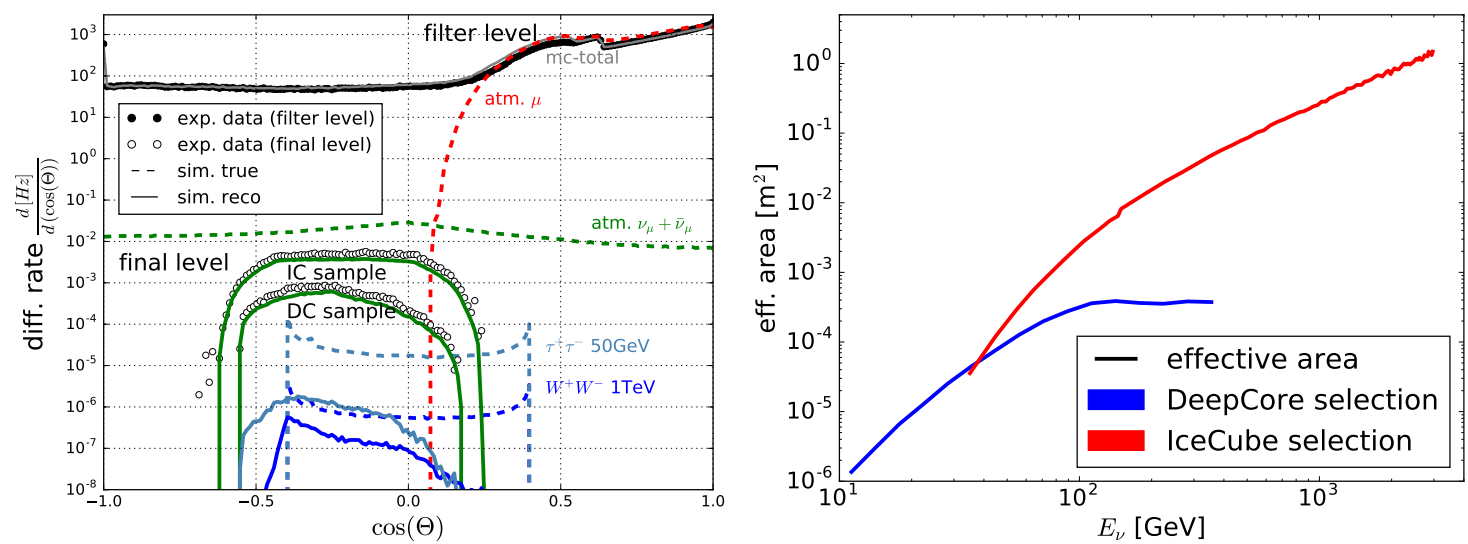

Figure 1: Right: simulation and data comparison as function of the cosine of zenith angle. Left: effective area as function of the reconstructed energy. 
Backgrounds of this analysis are atmospheric neutrinos and muons. The CORSIKA package [7] is used to calculate the interaction of comic rays with the Earths atmosphere for the atmospheric $\mu$ background. For the atmospheric $v$ background, we used neutrino-generator (NuGen) and GENIE respective for energies above and below $150 \mathrm{GeV}$.

\subsection{Event selection and Analysis}

We define two events selections optimized to different neutrino energy ranges. The first one referred to as DeepCore (DC), selects events that interact in the DeepCore detector, while the second sample, classified as IceCube (IC), sample focuses on more energetic neutrino events. To point events back to the Sun we optimize for muon neutrinos. We only select upgoing events and utilize the Earth as a filter against atmospheric muons $\mu$, this analysis mainly is focused on the upgoing events that starts below the detector and travel upwards through it.

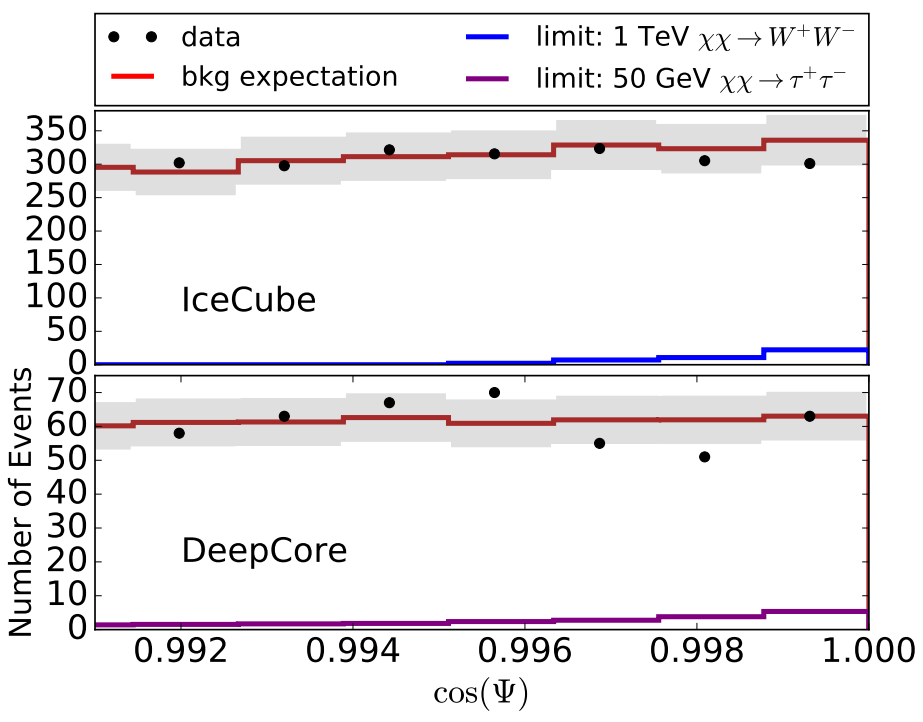

Figure 2: Events distribution for cosine of angular distance from the Sun. Black dots show the reconstructed data at the corresponding direction. The red lines are background expectation from MC simulation and gray regions represent statistical uncertainties on the background expectation. Blue $\left(\chi \chi \rightarrow W^{+} W^{-}\right)$and $\operatorname{purple}\left(\chi \chi \rightarrow \tau^{+} \tau^{-}\right)$lines are the expected solar WIMPs annihilation events in IceCube and DeepCore, respectively.

IC sample was optimized for neutrino events with energies above $\sim 100 \mathrm{GeV}$.

Figure 1 shows differential rates backgrounds at filter and final selection cut level for IC and DC samples. The background predictions are compared to observed event rates and signal expectations at current bounds. The points represent the data and lines show the simulation expectations. Dotted lines show the simulation prediction before the event selection. The red dotted line represents simulated atmospheric $\mu$ and green dotted line shows simulated atmospheric $v_{\mu}$. Downgoing events are dominated by atmospheric $\mu$ as without the Earth veto effect. The light blue and dark blue lines are the predicted neutrino flux from solar WIMP annihilations in the Sun respective for $\chi \chi \rightarrow \tau^{+} \tau^{-}$and $\chi \chi \rightarrow W^{+} W^{-}$. After the event selection, we get green solid lines for the simulation and white dots as data. In the right of figure 1 the effective area for the IC and DC event selections are shown.

Figure 2 shows the distribution of neutrino events with respect to the cosine angular separation of the Sun for IceCube (top) and DeepCore (bottom) samples. We estimate WIMP-proton cross section limits with an unbinned maximum likelihood method. For $\mathrm{N}$ events with $n_{s}$ signal events, the likelihood function is: 


$$
L\left(n_{S}\right)=\prod_{N}\left(\frac{n_{s}}{N} S_{i}+\left(1-\frac{n_{s}}{N}\right) B_{i}\right)
$$

$S_{i}$ and $B_{i}$ are probability density functions(p.d.f.) for signal and background respectively and depend on the reconstructed energy and position of the events. Each p.d.f. can be written as

$$
\begin{aligned}
S_{i}\left(\vec{x}_{i}, t_{i}, E_{i} ; m_{\chi}, c_{\chi}\right) & =K\left(\left|\vec{x}_{i}-\overrightarrow{x_{\odot}}\right|, k_{i}\right) \times \varepsilon_{m_{\chi}, c_{\chi}}\left(E_{i}\right) \\
B_{i}\left(\vec{x}_{i}, E_{i}\right) & =D\left(\delta_{i}\right) \times P\left(E_{i} \mid \phi_{\text {atm }}\right)
\end{aligned}
$$

The energy part of signal p.d.f. is dependent on the WIMPs mass $\left(m_{\chi}\right)$ and capture coefficient $\left(c_{\chi}\right) . K$ represents the angular distribution of the signal with the concentration factor, $k_{i}$ and is given by the Fisher-Binghan distribution [8]. The background p.d.f. consists of the declination dependence $(D)$ and the distribution $(P)$ of the energy estimator, $E$ in the event sample.

\subsection{Results}

There is no significant excess of events in the direction of the Sun. Assuming a local DM density of $0.3 \mathrm{GeV} / \mathrm{cm}^{3}$, a standard Maxwellian halo velocity distribution and the Standard Solar Model, we calculate the upper limit of the spin-dependent WIMP-proton cross section in IceCube (figure 3). We include 12$35 \%$ systematic uncertainties dependent on the

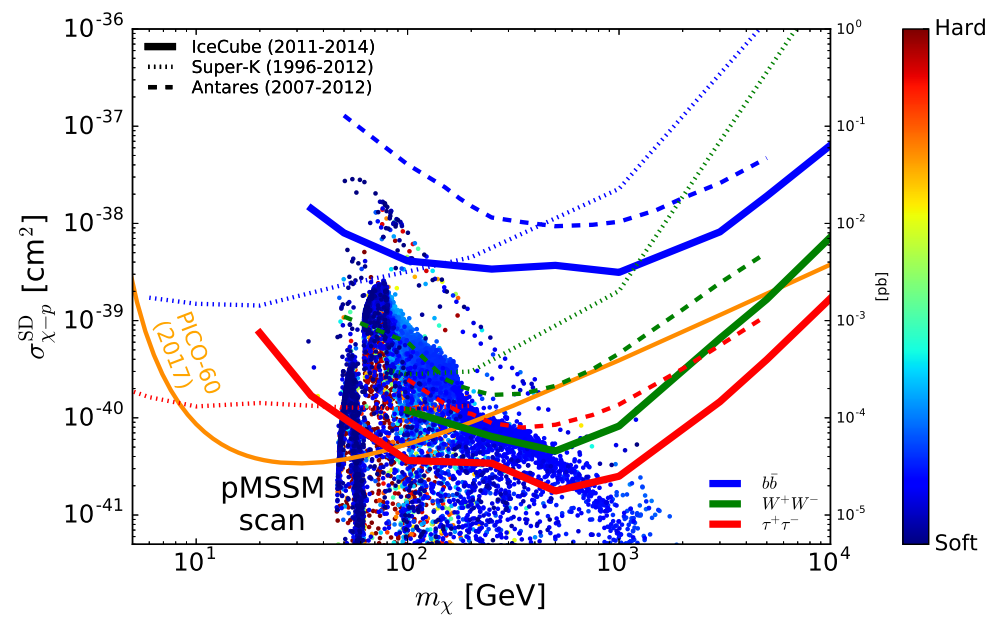

Figure 3: WIMP-proton spin dependent cross section with 3 years data of IceCube. Each color represents a different annihilation channel; blue $b \bar{b}$, green $-W^{+} W^{-}$, red $-\tau^{+} \tau^{-}$. As the reference limits, we marked Super$\mathrm{K}$ (dotted) and Antares (dashed) limit and from the direct detection PICO-60 $\mathrm{C}_{3} \mathrm{~F}_{8}$ (orange) [10].

WIMPs masses. IceCube limits are the most stringent above $\sim 80 \mathrm{GeV}$ (thick solid lines). Our limit excludes the models from a scan of $\sim 500$ million points in the 19 parameters of the phenomelogical minimally super-symmetic standard model (pMSSM) calculated by micrOMEGAs [9].

\section{All-flavor search for solar WIMPS}

The denser low energy infill, DeepCore, considerably improves neutrino detection with energies below $100 \mathrm{GeV}$ due to the higher sensor granularity and the veto capacity of the surrounding IceCube strings. IceCube can detect all flavors of active neutrinos through Cherenkov light emission from secondary particles created when a neutrino interacts in the ice. The reconstruction of electron and tau neutrino interactions, leaving cascade-like signatures in the detector, is more challenging than the reconstruction of track-like signatures. However, all-neutrino searches have 
become in focus recently. The reasons are obvious [11]: the measured flux is enhanced, the neutrino energies may be determined to a better precision, backgrounds from atmospheric $v_{\mathrm{e}}$ and $v_{\tau}$ are smaller and cosmic ray muons tend to be rejected better by requiring that the events have a cascade-like signature.

\subsection{Analysis}

An exploratory study of IceCube's sensitivity to WIMP annihilations in the center of the Sun with an analysis that is sensitive to all flavors of neutrinos was performed on data taken with the finalized configuration in the 2011 season. For the simulation of a WIMP-induced neutrino signal, the WimpSim package [6] was used which takes care of neutrino generation, propagation and oscillations.

Unlike the extended tracks caused by muons from chargedcurrent (CC) $v_{\mu}$ events, $v_{\mathrm{e}}$ and $v_{\tau}$

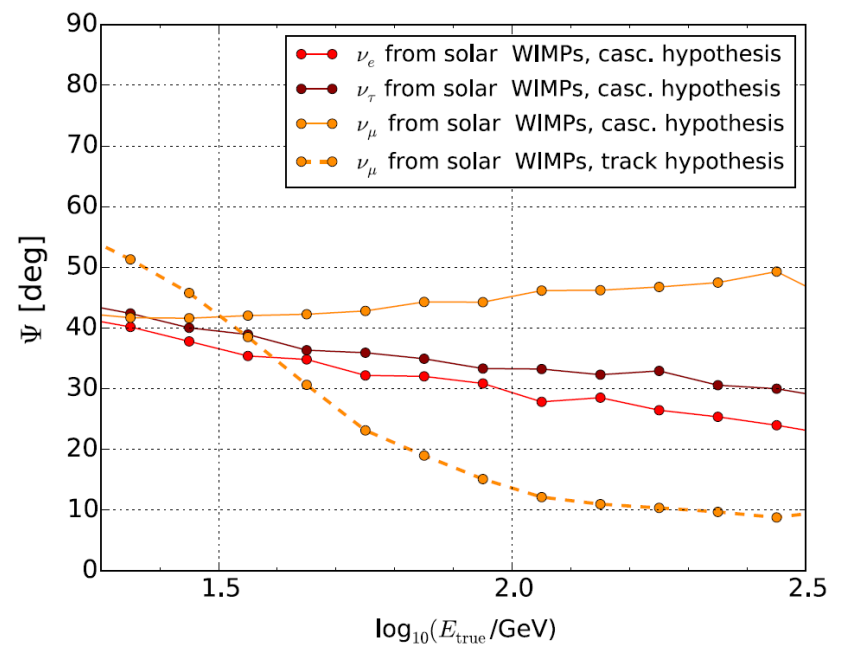

Figure 4: Median angular distance between simulated and reconstructed direction at the final selection level. Solid lines correspond to a cascade reconstruction while the dashed line represent a reconstruction assuming a track hypothesis.

leave an almost spherical pattern of hit DOMs in the detector. The $\mathrm{e}^{ \pm}$produced in CC $v_{\mathrm{e}}$ interactions are subject to successive bremsstrahlung energy losses and lead to electromagnetic cascades. $v_{\tau}$ interactions and $\tau$ decays predominantly produce hadronic cascades, as do neutral current interactions from all neutrino flavors. While the energy reconstruction benefits from the confined event signature, a good directional reconstruction of the spherically shaped cascade events demands significant computing resources and also an excellent description of the ice properties. Energy $E$, position and orientation are estimated [12] by minimizing the negative log-likelihood $-\ln L=\sum_{i} k_{i} \ln \left(E \Lambda_{i}+\rho_{i}\right)-\left(E \Lambda_{i}-\rho_{i}\right)-\ln k_{i}$ !, where $\rho_{i}$ is the expected number of noise photons and $k_{i}$ is the observed number of photons in a time bin $i$.

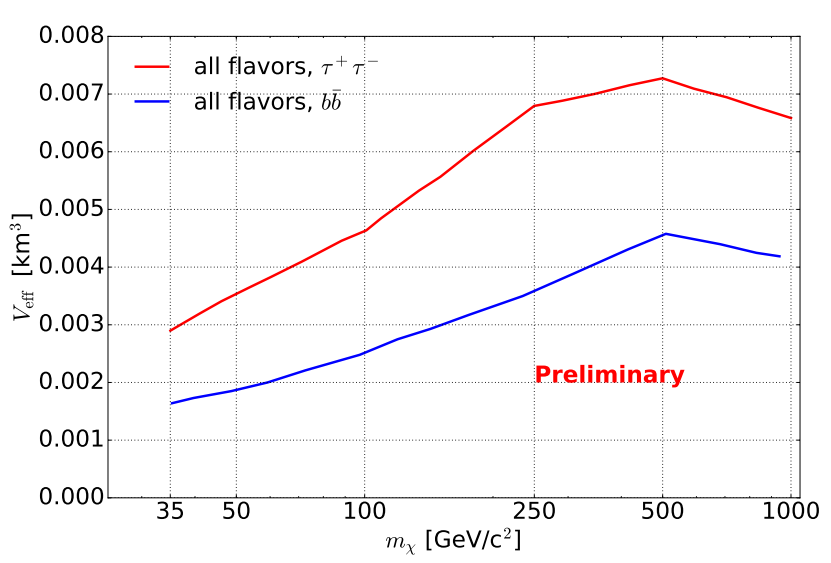

Figure 5: Final level effective volume as function of WIMP mass for soft $(b \bar{b})$ and hard annihilation $\left(\tau^{+} \tau^{-}\right)$channels.
The number of photons per unit energy for an assumed orientation and vertex $\Lambda_{i}$ incorporates detailed information on the position dependent absorption and scattering of photons in the ice. This information is available in the form of splinefitted tables obtained from a photontracking simulation using a ray tracing algorithm modeling scattering and absorption. When iterating the minimization chain (in this analysis 32 times) and optimizing minimiza- 
tion parameters, the resulting angular resolutions are similar to the ones seeded by the true direction and vertex.

The energy dependent median spatial angle resolution is shown in Fig. 4. Since no reliable flavor identification is possible on an event by event basis without substantial efficiency losses, the cascade hypothesis is used for all events. It is evident from Fig. 4 that the cascade reconstruction is actually superior to the track hypothesis reconstruction for all all neutrino species with energies below $35 \mathrm{GeV}$.

Individual event resolutions may vary from the average resolution dependent on the event's exact topology and the amount of light deposited in the detector volume. Since event-based resolutions allow for a reconstruction quality based event weighting, a resolution estimator, based on the Cramer-Rao upper bound on the variance, was coded [4].

In a series of selection levels, the dominant background from atmospheric muons is reduced, followed by filters that effectively remove noise clusters and coincidences,

\begin{tabular}{|c|c|c|c|c|c|c|c|c|c|}
\hline & \multicolumn{4}{|c|}{$b \bar{b}$} & \multicolumn{3}{|c|}{$\tau^{+} \tau^{-}$} & \multicolumn{3}{|c|}{$W^{+} W^{-}$} \\
\hline $\begin{array}{c}m_{\chi} \\
{\left[\mathrm{GeV} / \mathrm{c}^{2}\right]}\end{array}$ & $\begin{array}{c}\varepsilon \\
{[\%]}\end{array}$ & $\begin{array}{c}\mu_{\mathrm{fit}}^{90 \%} \\
{[\mathrm{events}]}\end{array}$ & $\begin{array}{c}\sigma_{\mathrm{SD}}^{\chi, p} \\
{[\mathrm{fb}]}\end{array}$ & $\begin{array}{c}\varepsilon \\
{[\%]}\end{array}$ & $\begin{array}{c}\mu_{\mathrm{fit}}^{90 \%} \\
{[\mathrm{events}]}\end{array}$ & $\begin{array}{c}\sigma_{\mathrm{SD}}^{\chi, p} \\
{[\mathrm{fb}]}\end{array}$ & $\begin{array}{c}\varepsilon \\
{[\%]}\end{array}$ & $\begin{array}{c}\mu_{\mathrm{fit}}^{90 \%} \\
{[\mathrm{events}]}\end{array}$ & $\begin{array}{c}\sigma_{\mathrm{SD}}^{\chi, p} \\
{[\mathrm{fb}]}\end{array}$ \\
\hline \hline 35 & 12.0 & 157 & 1.8 & 10.2 & 145 & 0.1 & - & - & - \\
\hline 50 & 10.6 & 141 & 2.0 & 8.1 & 137 & 0.1 & - & - & - \\
\hline 100 & 7.9 & 139 & 2.7 & 4.3 & 103 & 0.1 & 4.7 & 106 & 0.22 \\
\hline 250 & 4.5 & 119 & 5.0 & 2.3 & 72 & 0.14 & 2.2 & 69 & 0.34 \\
\hline 500 & 3.3 & 113 & 10 & 1.6 & 54 & 0.29 & 1.5 & 47 & 0.72 \\
\hline 1000 & 2.0 & 77 & 23 & 1.2 & 40 & 0.75 & 1.1 & 39 & 2.6 \\
\hline
\end{tabular}

Table 1: Selected final level efficiencies $(\varepsilon)$ with respect to level 2, best fit sensitivity on number of signal events and spin-dependent WIMP cross section limits, $\sigma_{\mathrm{SD}}^{\chi, p}$ (excluding systematic uncertainties).

Finally, a set of Boosted Decision Trees (BDTs) using 12 variables, including reconstructed direction, energy and vertex, reconstruction quality parameters as well as veto and geometrical quantities, was trained to discriminate signal-like from background-like events [4]. The BDT score cut was optimized to provide the best sensitivity for a given WIMP mass and annihilation channel. Signal efficiencies $\left(\varepsilon\right.$ ) for WIMPs with masses below $250 \mathrm{GeV} / \mathrm{c}^{2}$ are listed in Table 1 .

For the distinction of signal and background events, a likelihood code [13] commonly employed in IceCube point source searches was adapted. The code, which includes event-based energy and angular uncertainty information, was altered substantially to work for cascade-shaped events with large angular uncertainties. For example, the signal PDF was expressed analytically by a von Mises-Fisher distribution and the background and energy PDFs were represented by a spline-smoothed two-dimensional histogram, where the binning was optimized automatically for each BDT score.

\subsection{Systematic uncertainties}

The likelihood analysis uses scrambled experimental data for the background level estimation, which therefore is not subject to atmospheric flux models, neutrino cross sections and the detector modeling. The sensitivities and upper limits, however, are calculated by injecting simulated signal 
events and as such depend on the correct modeling of the detector response and particle physics uncertainties, such as oscillation parameters and neutrino cross section in the transition region to deep inelastic scattering. Astrophysical uncertainties, like the local dark matter density and local velocity, are also important.

The dominant detector related uncertainty, especially at low energies, is the absolute DOM light detection efficiency of roughly $10 \%$. Its effect on the signal was assessed by the productin of datasets with artificially lowered and increased efficiencies, leading to asymmetric uncertainty estimates. The total uncertainty, including effects from the ice model, neutrino cross sections and oscilla-

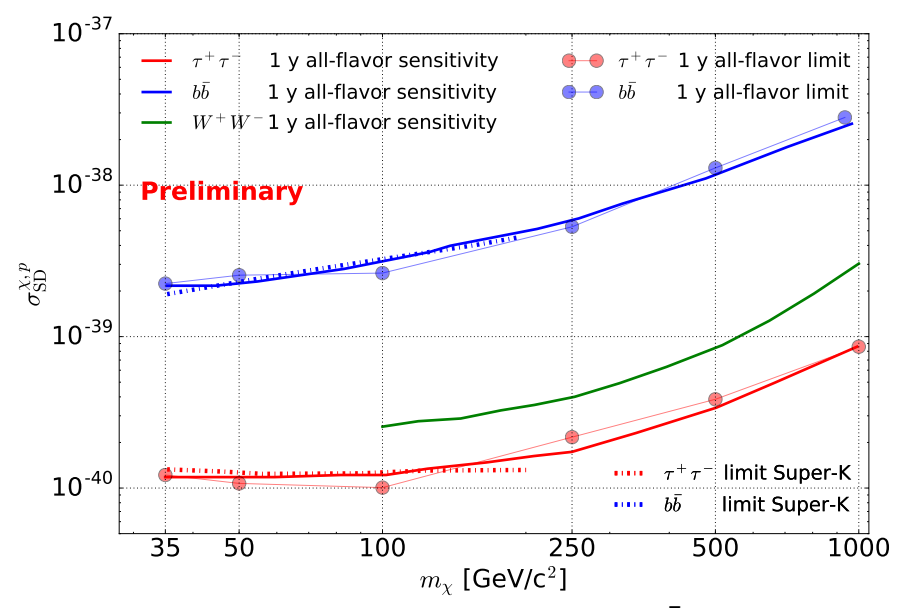

Figure 6: Upper limits and sensitivities for the $b \bar{b}$ and $\tau^{+} \tau^{-}$channels. tions,ranges between $16.6 \%$ and $21 \%$, slightly depending on the annihilation channel and assume WIMP mass. For this study a local dark matter density of $\rho_{0}=0.30 \mathrm{GeV} / \mathrm{c}^{2} / \mathrm{cm}^{3}$ was adapted. The results can be easily rescaled as the limits scale inversely with the assumed matter density (larger densities yield sensitivities to smaller WIMP scattering cross sections).

\section{Solar WIMP sensitivity for IceCube-Gen2/PINGU}

IceCube-Gen2/PINGU is a proposed extension of IceCube envisioned to include the deployment of 20 densely instrumented strings in the central region of IceCube. IceCube-Gen2/PINGU is expected to significantly improve the sensitivity for WIMP masses in the energy range between 5 and $50 \mathrm{GeV}$. Building on the experience gained with DeepCore analyses we perform a straight-
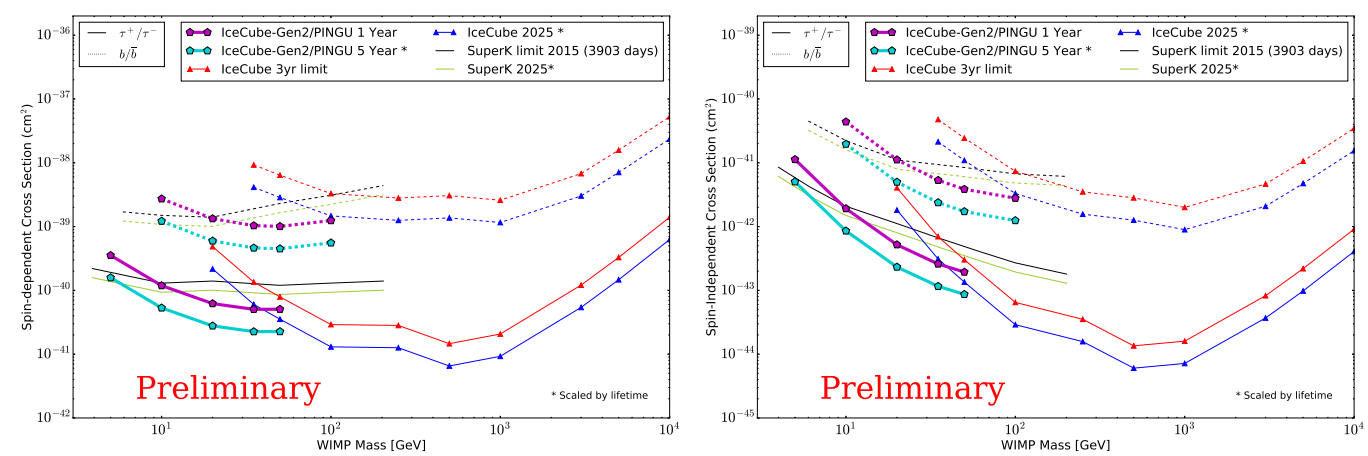

Figure 7: Sensitivity of the $26 \times 192$ IceCube-Gen2/PINGU baseline geometry to $\sigma_{p, S D}$ (left figure) and $\sigma_{p, S I}$ (right figure) for hard (solid lines) and soft (dashed lines) annihilation channels over a range of WIMP masses for livetimes of one (magenta) and five (green) years. The sensitivities are compared to the present IceCube limits [3] and limits from Super-K [14], as well as limits projected to the year 2025 to compare to the IceCube-Gen2/PINGU sensitivities. 
forward event-based Monte Carlo (MC) study, using the $26 \times 192$ IceCube-Gen2/PINGU baseline geometry. We use standard event selection criteria and apply a fixed search window around the position of the Sun with a half-cone opening angle of $10^{\circ}$. Figure 7 shows the sensitivity for IceCube-Gen2/PINGU combined with the scaled currently best published IceCube limit.

\section{Summary and Outlook}

IceCube has searched for dark matter captured in the Sun and produced some of the most stringent limits on the spin-dependent WIMP-nucleon scattering cross section. A new analysis using all neutrino flavors has significantly improved sensitivities for WIMP masses below $50 \mathrm{GeV}$. While high-energy neutrinos $(>1 \mathrm{TeV})$ cannot escape the center of the Sun due to absorption, energetic neutrinos could be produced in scenarios with secluded dark matter. In such a scenario DM annihilates into long lived mediators which can escape from the Sun, and could decay into neutrinos $[15,16]$. Cosmic-ray interactions in the solar atmosphere could also produce energetic neutrinos and are lately of high theoretical interest [17] [18]. Solar atmospheric neutrinos pose a natural background to solar dark matter searches. Analyses efforts in IceCube have started to target energetic neutrinos from the Sun [19]. Lastly with the Gen2 extension to the IceCube detector the sensitivity to dark matter masses below $100 \mathrm{GeV}$ can be significantly improved.

\section{References}

[1] IceCube Collaboration, M.G. Aartsen et al., JINST 12 (2017) P0301.

[2] G. Jungman, M. Kamionkowski, K. Griest, Phys. Rept. 267 (1996) 195.

[3] IceCube Collaboration, M.G. Aartsen et al., Eur. Phys. J. C77 (2017) 146

[4] IceCube Collaboration, Klaus Wiebe and Anna Steuer, POS ( ICRC2015) 1224 (2015).

[5] P. Gondolo, J. Edsjö, P. Ullio, L. Bergström,M. Schelke and E.A. Baltz, JCAP 07 (2004) 008.

[6] M. Blennow, J. Edsjö and T. Ohlsson, JCAP 01 (2008) 021.

[7] D. Heck et al., (Forschungszentrum Karlsruhe) Tech. Rep. FZKA-6019 (1998).

[8] J.T. Kent, J. Royal. Stat. Soc. 44 (1982) 71.

[9] G. Belanger et al., Comput. Phys. Commun. 185 (2014) 960.

[10] PICO Collaboration, C.Amole et al., Phys. Rev. Lett 118 (2017) 251301.

[11] C. Rott, T. Tanaka and Y. Itow, JCAP 1109 (2011) 029.

[12] IceCube Collaboration, M. G. Aartsen et al., JINST 9 (2014) P03009.

[13] IceCube Collaboration, Stephan Coenders PoS ( ICRC2015) 1047 (2015).

[14] Super-Kamiokande Collaboration, K. Choi et al., Phys. Rev. Lett. 114 (2015) 141301.

[15] N. F. Bell and K. Petraki, JCAP 1104 (2011) 003.

[16] P. Meade, S. Nussinov, M. Papucci and T. Volansky, JHEP 1006 (2010) 029.

[17] C.A. Argüelles, G. de Wasseige, A. Fedynitch and B.J.P. Jones, [ast ro-ph/1703.07798].

[18] Kenny C. Y. Ng, John F. Beacom, Annika H. G. Peter and C. Rott, [astro-ph/1703. 10280].

[19] IceCube Collaboration, Seongjin In and Carsten Rott POS ( ICRC2017) 965 (these proceedings). 